\title{
Beam division multiple access for millimeter wave massive MIMO: Hybrid zero-forcing beamforming with user selection
}

\author{
Hong Son Vu ${ }^{1}$, Kien Trung Truong ${ }^{2}$, Minh Thuy Le ${ }^{1}$ \\ ${ }^{1}$ School of Electrical Engineering, Hanoi University of Science and Technology, Hanoi, Vietnam \\ ${ }^{2}$ Fulbright University Vietnam, Ho Chi Minh City, Vietnam
}

\begin{tabular}{l} 
Article Info \\
\hline Article history: \\
Received Dec 29, 2020 \\
Revised Jul 16, 2021 \\
Accepted Aug 2, 2021 \\
\hline
\end{tabular}

Keywords:

Hybrid beamforming

Massive MIMO

mmWave

\begin{abstract}
Massive multiple-input multiple-output (MIMO) systems are considered a promising solution to minimize multiuser interference (MUI) based on simple precoding techniques with a massive antenna array at a base station (BS). This paper presents a novel approach of beam division multiple access (BDMA) which BS transmit signals to multiusers at the same time via different beams based on hybrid beamforming and user-beam schedule. With the selection of users whose steering vectors are orthogonal to each other, interference between users is significantly improved. While, the efficiency spectrum of proposed scheme reaches to the performance of fully digital solutions, the multiuser interference is considerably reduced.
\end{abstract}

This is an open access article under the CC BY-SA license.

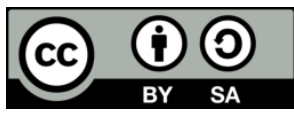

\section{Corresponding Author:}

Minh Thuy Le

Department of Instrument and Industrial Informatics, School of Electrical Engineering, Hanoi University of Science and Technology

No. 1 Dai Co Viet Street, Hai Ba Trung District, Hanoi, Vietnam

Email: thuy.leminh@hust.edu.vn

\section{INTRODUCTION}

Nowadays, the increasingly high number of mobile users has put pressure on current communication systems adopting such techniques as time-division multiple access (TDMA) and frequencydivision multiple access (FDMA). The overload is especially intensified in the new 5th Generation system. In order to tackle the problem of massive users and increase access capacity, beam division multiple access (BDMA) has been proposed to the system [1], [2]. The BDMA scheme basically consists of three steps: i) base station (BS) acquires channel coupling matrices (CCMs) from all user equipments (Ues) in its cell, ii) user selection based on CCMs, then beams at BS are group into separated sub-sets, and iii) transmitting pilot and data in the uplink and downlink. A selecting scheme within non-overlapping beams is proposed to BDMA based on decomposition of the MU-MIMO channels into multiple single-user MIMO channels [1]. As a result, the overhead of channel estimation, as well as the processing complexity are improved significantly at transceivers. Meanwhile, Jiang et al. [2] apply lyapunov-drift optimization framework for joint user scheduling and beam selection method, thereby obtaining an optimal scheduling policy in a closed form.

The average channel capacity of wireless communication systems is heavily affected by fading environments [3]-[6]. Especially, channel capacity degrades significantly it'sin the fading correlations enivroments [7]. Massive multiple-input multiple-output (MIMO) techniques, for example, hybrid beamforming architecture, were developed to attain the improving channel capacity of system in rich scattering environments. Hybrid beamforming was deployed widely for millimeter wave (mmWave) massive MIMO transmissions [8]-[11]. This architecture is considered the most effective solution to power consumption and production costs problems of fully-digital architecture based on an additional layer of analog phase shifters [12]. Moreover, the hybrid beamforming also achieves efficiency approximating to the 
performance of digital solutions but at a much lower cost [13]. Transmit beamforming strategies for the cellular downlink become the attractive topic [14]-[19]. There are several previous works proposed such efficient beamforming strategies as zero-forcing beamforming (ZFBF) [14], [20]-[22], orthogonal beamforming (OBF) [23], [24], and orthogonal random beamforming (ORBF) [4]. If the number of mobile stations is higher than the number of antenna, user selection technique is essential to selects a group of users for simultaneous transmission [25]. Works in [20], [21] proposed two selection algorithms for MIMO which are semi-orthogonal user selection (SUS) and greedy user selection (GUS). In [21], by combining ZFBF with SUS (ZFBF-SUS), the optimal sum rate could be achieved asymptotically with fully-digital, as the number of users approaches infinity.

In this work, we study a low-complexity downlink-beamforming algorithm for step 2 in BDMA scheme such that maximize sum capacity for the practical case when the number of downlink users exceeds the number of antennas. Therefore, we propose a multiple access scheme that employs BMDA design with hybrid beamforming structure and simple user-beam schedule, which not only reaches asymptotically optimal in the sum-rate, but the average multiuser interference (MUI) energy can be minimized. In each time slot, before the antenna weight vectors are updated base on ZFBF, the base station will select those mobile stations such that the interference between them is minimal. To be specific, the system aims at opportunistically schedule users whose steering vectors are as orthogonal as possible to each other. Subsequently, this scheduling scheme will reduce the average multiuser interference MUI energy over all the mobile stations (MSs).

The content of paper is organized as 5 sections. In section 2, we present the problem formulation, and $\mathrm{ZF}$ with user selection algorithm is considered in section 3. Results and discussions are given in section 4 to demonstrate the effectiveness of our algorithm. Some conclusions are given in the last section. Notation: uppercase boldface letters and lowercase boldface are described correspondingly for matrices and vectors. The operators $\|\|,.(.)^{T},(.)^{H}$ and |. | denote norm, transpose, Hermitian conjugate and modulus, respectively.

\section{PROBLEM FORMULATION}

Generally, the hybrid beamforming architecture could be divided into fully connected and partially connected one [26]. While each radio-frequency (RF) chain of a fully connected architecture is mapped with all antenna elements by phase shifter network, a partially connected hybrid transmitter connects each RF chain to an antenna sub-array. In this paper, we conducted our proposed algorithm with fully connected hybrid architecture as shown in Figure 1.

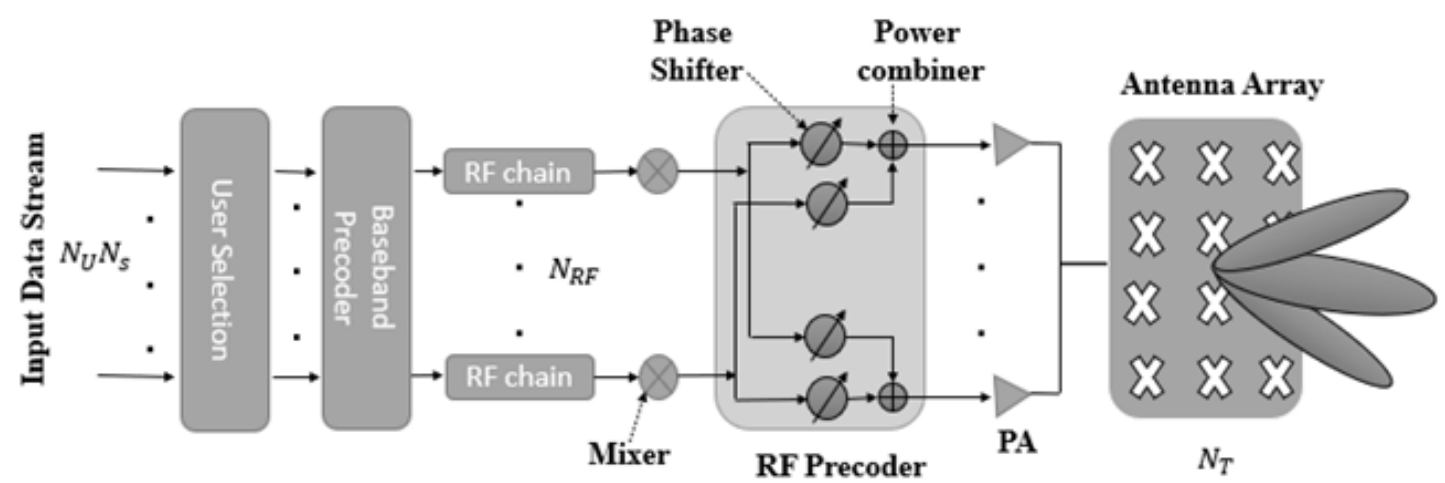

Figure 1. A fully connected hybrid beamformer

We consider a massive mmWave MIMO system with $\mathrm{N}_{\mathrm{T}}$ transmitted antennas and $\mathrm{N}_{\mathrm{RF}} \mathrm{RF}$ chains at BS serving $\mathrm{N}_{U}$ mobile station (MS) with $\mathrm{N}_{R}$ received antenna at each one. In this work, we concentrate on a single block transmitted to $\mathrm{N}_{\mathrm{U}}$ users. The resulting signal $\mathrm{x}$ of dimension $\mathrm{N}_{\mathrm{T}} \times 1$ after hybrid beamforming system is modeled as in (1):

$$
\mathrm{x}=\mathrm{F}_{R F} \times \mathrm{F}_{B B} \times \mathrm{s}=\mathrm{F} \times \mathrm{s}
$$

where $F_{R F}=\left[f_{1, R F}, f_{2, R F}, \ldots, f_{N_{R F}, R F}\right]$ is analog beamforming matrix with $f_{u, R F} \in \mathbb{C}^{N_{T} \times 1}$ being the $u$-th analog beamforming vector, the digital beamforming matrix $\mathrm{F}_{\mathrm{BB}}=\left[\mathrm{f}_{1, \mathrm{BB}}, \mathrm{f}_{2, \mathrm{BB}}, \ldots, \mathrm{f}_{\mathrm{N}_{\mathrm{RF}}, \mathrm{BB}}\right]$ with $\mathrm{f}_{\mathrm{u}, \mathrm{BB}} \in \mathbb{C}^{\mathrm{N}_{\mathrm{S}} \times 1}$ is the 
digital beamforming vector for the $\mathrm{u}$-th user, and $\mathrm{s}_{\mathrm{U}}$ is the block data of $\mathrm{u}$-th user. The received signal of the u-th user can be expressed as (2):

$$
y_{u}=\underbrace{\mathrm{H}_{u} \cdot \mathrm{f}_{u, R F} \cdot \mathrm{f}_{u, B B} \cdot s_{u}}_{\text {desired }}+\underbrace{\sum_{i \neq u} \mathrm{H}_{u} \cdot \mathrm{f}_{u, R F} \cdot \mathrm{f}_{i, B B} \cdot s_{i}}_{\text {interferece }}+\underbrace{n_{u}}_{\text {noise }}
$$

where $\mathrm{y}_{u} \in \mathbb{C}^{N_{R} \times 1}, \mathrm{H}_{u} \in \mathbb{C}^{N_{R} \times N_{T}}$ is the MIMO channel matrix between the transmitter and the u-th receiver, and $\mathrm{n}_{\mathrm{u}} \in \mathbb{C}^{N_{R} \times 1}$ is the complex additive white gaussian noise with zero mean and variance of $\sigma^{2}$ for $\mathrm{u}$-th user. At $\mathrm{u}$-th MS, the beamforming weight vector $\mathrm{w}$ combined the $\mathrm{RF} \mathrm{w}_{R F}$ and baseband $\mathrm{w}_{B B}$ precoding vectors. The decoded signal of the $\mathrm{u}$-th receiver is given by (3):

$$
\tilde{s}_{u}=\mathrm{w}_{u}^{H} \mathrm{H}_{u} \mathrm{f}_{u, R F} \mathrm{f}_{u, B B} s_{u}+\mathrm{w}_{u}^{H} \tilde{n}_{u}
$$

where $\tilde{n}_{u}$ is the sum of complex additive white Gaussian noise (AWGN) and interference from other users. The u-th user's channel model of mmWave systems can be modeled as in (4) [27], [28]:

$$
\mathrm{H}_{u}=\sqrt{\frac{N_{T} N_{R}}{N_{L}}} \sum_{l=1}^{N_{L}} \alpha_{u, l} \cdot \mathrm{a}_{R}\left(\phi_{u, l}^{r}, \varphi_{u, l}^{r}\right) \cdot \mathrm{a}_{T}^{H}\left(\phi_{u, l}^{t}, \varphi_{u, l}^{t}\right)
$$

where $\mathrm{N}_{\mathrm{L}}$ is the number of scatters of the u-th user's channel, $\alpha_{\mathrm{u}, \mathrm{l}}$ is the complex path gain, $\mathrm{a}_{\mathrm{R}}\left(\phi_{\mathrm{u}, \mathrm{l}}^{\mathrm{r}}, \varphi_{\mathrm{u}, \mathrm{l}}^{\mathrm{r}}\right)$ and $\mathrm{a}_{\mathrm{T}}\left(\phi_{\mathrm{u}, 1}^{\mathrm{t}}, \varphi_{\mathrm{u}, \mathrm{l}}^{\mathrm{t}}\right)$ are received and transmitted steering vectors. In this work, a $\mathrm{N}_{\mathrm{h}} \times \mathrm{N}_{\mathrm{w}}$ uniform planar array (UPA) is used, the steering vector can be rewritten [29]:

$$
\mathrm{a}(\phi, \varphi)=\frac{1}{\sqrt{N_{T}}}\left[1, e^{j k d(\sin \phi \sin \varphi+\cos \varphi)}, \ldots, e^{j k d\left(\left(N_{h}-1\right) \sin \phi \sin \varphi+\left(N_{w}-1\right) \cos \varphi\right)}\right]^{T}
$$

where $k=\frac{2 \pi}{\lambda}$ is the wavenumber and $d$ is the distance between two adjacent element antennas. Then, the signal-to-interference plus noise ratio (SINR) of user $u^{\text {th }}$ is (6),

$$
\operatorname{SINR}_{u}=\frac{\left|\mathrm{H}_{u} \cdot \mathrm{f}_{u, R F} \cdot \mathrm{f}_{u, B B}\right|^{2}}{\sum_{i \neq u}\left|\mathrm{H}_{u} \cdot \mathrm{f}_{u, R F} \cdot \mathrm{f}_{i, B B}\right|^{2}+\sigma^{2}}
$$

The beamforming cost function can now be formulated as (7):

$$
\max _{\mathrm{F}_{R F}, \mathrm{~F}_{B B}} \sum_{u=1}^{N_{U}} \log _{2}\left(1+\operatorname{SIN} R_{u}\right) \text { subject to }\left\|\mathrm{F}_{\mathrm{RF}} \cdot \mathrm{F}_{B B}\right\|^{2}=1
$$

\section{PROPOSED ZF WITH GREEDY ALGORITHM}

\subsection{Proposed algorithm}

We assumed that BS has the perfect channel state information (CSI). Therefore, angle of arrival (AoA) and angle of departure (AoD) information $\left\{\phi_{u}^{r}, \varphi_{u}^{r}, \phi_{u}^{t}, \varphi_{u}^{t}\right\}$ is perfectly known. As a result, optimal analog beamforming vector of $\mathrm{u}$-th user could be computed (8):

$$
\mathrm{f}_{u, R F}^{*}=\mathrm{a}_{T}\left(\phi_{u}^{t}, \varphi_{u}^{t}\right), u=1 \div N_{U}
$$

The optimization problem in (7) is rewritten (8):

$$
\max _{\mathrm{F}_{B B}} \sum_{u=1}^{N_{U}} \log _{2}\left(1+S I N R_{u}\right) \text { subject to }\left\|\mathrm{F}_{R F}^{*} \cdot \mathrm{F}_{B B}\right\|^{2}=1
$$

From (3), set $\mathrm{g}_{u}^{H}=\mathrm{w}_{u}^{H} \times \mathrm{H}_{u} \times \mathrm{f}_{u, R F}^{*}$, then $G=\left[\mathrm{g}_{1}, \mathrm{~g}_{2}, \ldots, \mathrm{g}_{N_{U}}\right]^{\mathrm{H}}$. According to a zero-forcing approach, in case of $N_{T}>N_{U}$, the optimal DBF matrix could be calculated as $\mathrm{F}_{B B}=\mathrm{G}^{\dagger}=\mathrm{G}^{\mathrm{H}}\left(\mathrm{GG}^{\mathrm{H}}\right)^{-1}$. Normalized 
baseband precoder $\mathrm{F}_{B B}$ is obtained $\mathrm{F}_{B B}^{*}=\frac{\mathrm{F}_{B B}}{\left\|\mathrm{~F}_{R F}^{*} \cdot \mathrm{F}_{\mathrm{BB}}\right\|}$. For $N_{T}<N_{U}$, to select subset users, we proposed a lowcomplexity algorithm, named ZF with user selection (ZFUS), as summarized in next.

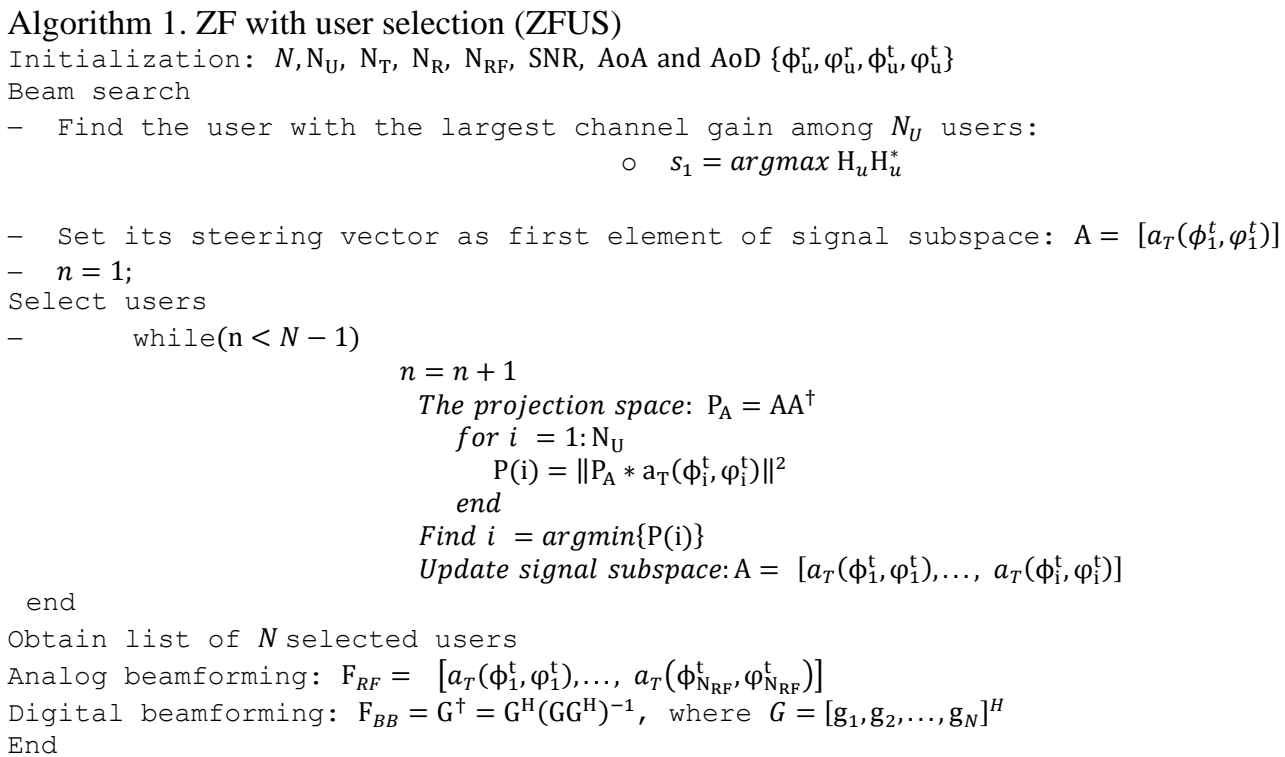

There are two main steps in user selection: beam search and select user. In the first phase, based on broadcast beam at BS side, we can find out UE with largest channel. Then, we set its steering vector $a_{T}\left(\phi_{1}^{t}, \varphi_{1}^{t}\right)$ as first element of signal subspace A. For the next phase, by comparing orthogonality between user's steering vector and projection space, the algorithm will select $(\mathrm{N}-1)$ users among the rest of $\left(\mathrm{N}_{\mathrm{U}}-1\right)$ users.

\subsection{Computational complexity analysis}

In this subsection, the computational complexity of the ZFUS algorithm is analyzed. The implementation of ZFUS consist of two phases: User selection and beamforming vector calculation. Because the latter requires only $N \times N$ matrix inversion to calculate beamforming weight, we focus on the complexity of user selection phase. In the beam search step, we need one $2 N_{T}$ matrix-matrix multiplication per user. With $N_{U}$ users, we need $2 N_{T} N_{U}$ matrix-matrix multiplication. The complexity of the selecting user step can be expressed as $\sum_{k=1}^{N} k\left(8 N_{T} k^{2}-N_{T} k+2\left(N_{U}-1\right) N_{T} k\right)=\sum_{k=1}^{N} k\left(8 N_{T} k^{2}+\left(2 N_{U}-3\right) N_{T} k\right)$. As a result, the number of computations of running the ZFUS algorithm is $2 N_{T} N_{U}+\sum_{k=1}^{N-1}\left(8 N_{T} k^{2}+\left(2 N_{U}-\right.\right.$ 3) $\left.N_{T} k\right)$.

\section{SIMULATION EVALUATION}

The performance of proposed ZFUS algorithms will be illustrated numerically in terms of the average MUI energy over all the MSs, system sum-rate capacity conditions used in our simulation are: i) the inner distance of two adjacent antennas is $0.5 \lambda$, ii) the number of total users $N_{U}=20$ users with a $2 \times 2$ UPA $\left(N_{R}=4\right)$ and the number of selected users $N=4$, and iii) the number of realizations is 500 for each experiment. Figure 2 shows the system sum-rate capacity versus SNR in $256 \times 16$ system with uniform planar array at both the transmitted and received side. It can be seen that the performance of ZFUS is approached to fully digital precoding system with a small gap. When compared to analog beamforming (ABF), Figure 2 shows that the ZFUS significantly improves the performance of system.

Next, we investigated the performance of three architectures following the number of base station antennas. Figure 3 shows that spectral efficiency (SE) values of three systems rise rapidly as the number of antennas increases from 16 to 250 elements. Though the SE of ZFUS is always higher than ABF about 4 $\mathrm{bps} / \mathrm{Hz}$, it could not reach asymptotically the fully digital system.

Finally, we study the Average of MUI energy over all the UTs versus number of BS antennas in Figure 4. It's clear that ZFUS can reduce MUI more effectively by selecting the user group with orthogonal steering vector to each other. While the MUI value of the ABF is always greater than $0 \mathrm{~dB}$, the ZFUS maintains the MUI value below $-15 \mathrm{~dB}$. As the number of antennas increases, both the MUI value of ZFUS and $\mathrm{ABF}$ tend to decrease. 


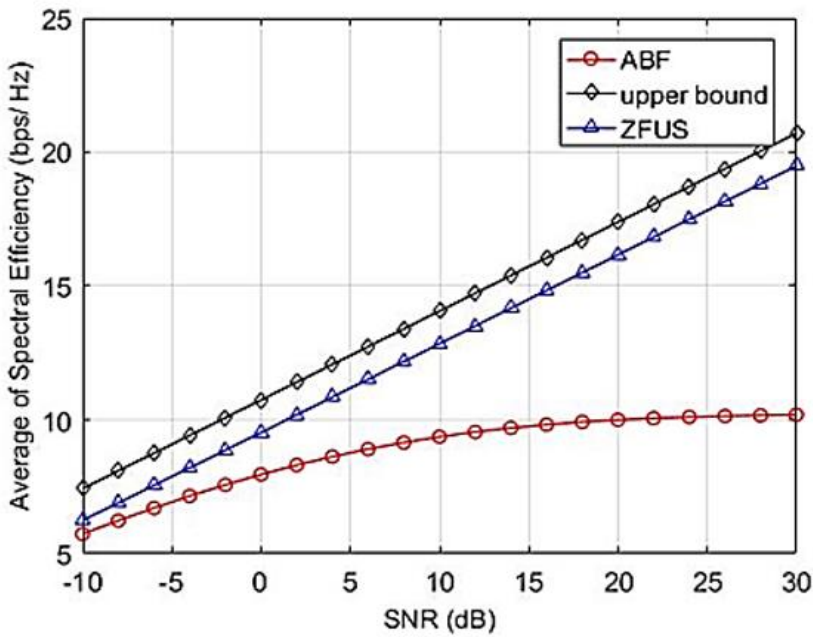

Figure 2. Comparison of sum-rate capacity versus SNR with $N_{-} T=16 \times 16$

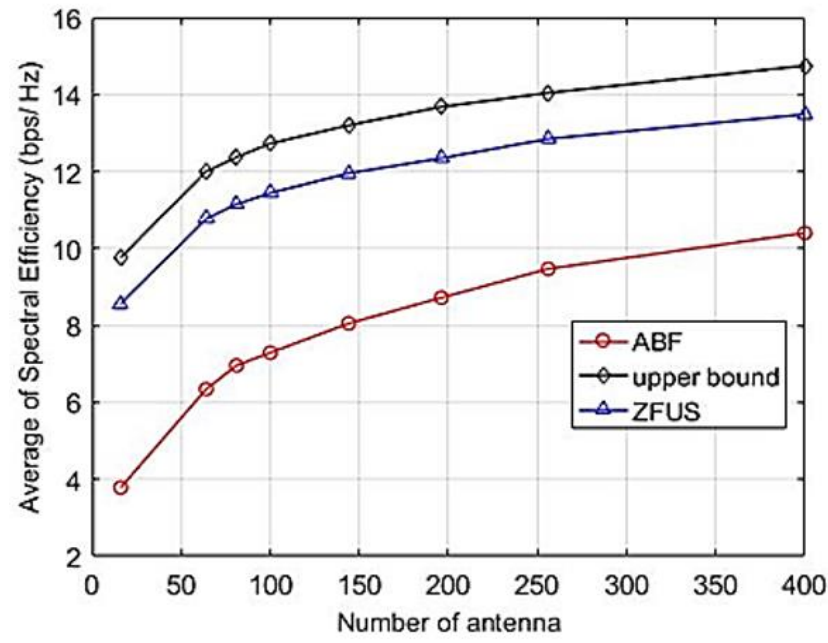

Figure 3. Comparison of sum-rate capacity versus number of BS antennas

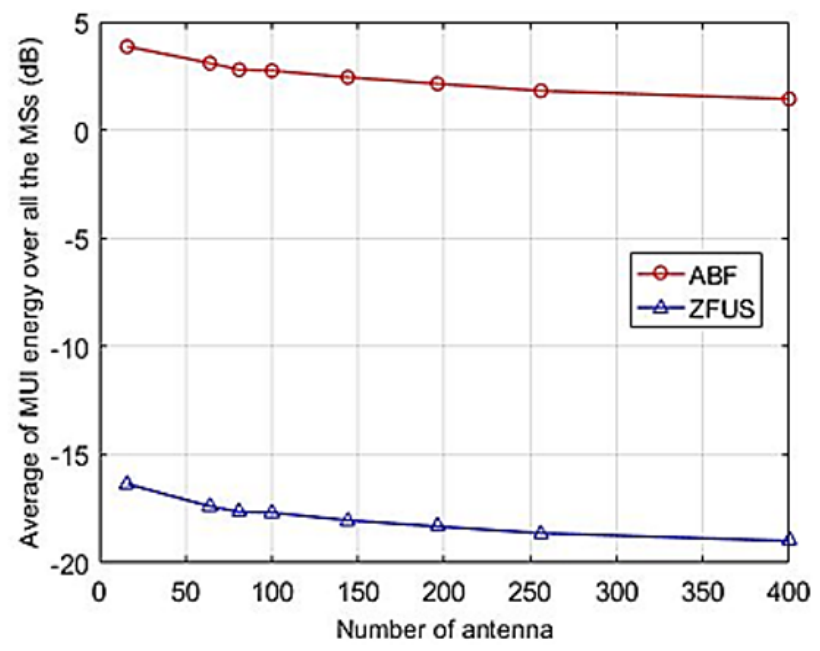

Figure 4. Average of multiuser interference at BS 
Compared to several relevant papers in Table 1, the performance of our proposed is better under the same architecture and SNR. To reduce the computational complexity, Singh and Ramakrishna [30] use subconnected structure and codebook method, this leads to decrease the spectral efficiency of system. In contrast, though works in [31], [32] deployed the fully structure and zero-forcing method same as our work, their spectral efficiency values are still lower than our work. It proves that the proposed user schedule help to improve the performance of system.

Table 1. The comparison of our proposed and previous works

\begin{tabular}{|c|c|c|c|c|}
\hline Paper & Architectures & Antenna Array & Algorithms & $\mathrm{SE}(\mathrm{b} / \mathrm{s} / \mathrm{Hz})$ \\
\hline$[30]$ & Sub-connected & $\begin{array}{c}N_{T}=2 \times 8 \\
N_{R}=2 \times 4 \\
N_{U}=1\end{array}$ & $\begin{array}{c}\text { ABF: codebook-based } \\
\text { DBF: a specified codebook of matrices }\end{array}$ & 10@SNR 10 dB \\
\hline [31] & Fully connected & $\begin{array}{c}N_{T}=64 \\
N_{R}=1 \\
N_{U}=2\end{array}$ & $\begin{array}{l}\text { ABF: Hermitian of downlink channel matrix } \\
\text { DBF: ZF }\end{array}$ & 1.37@SNR 0 dB \\
\hline$[32]$ & Fully connected & $\begin{array}{c}N_{T}=64 \\
N_{R}=16 \\
N_{U}=4\end{array}$ & $\begin{array}{l}\text { ABF: beam steering codebook } \\
\text { DBF: ZF based on perfect CSI }\end{array}$ & 6.8@SNR 0 dB \\
\hline My Work & Fully connected & $\begin{array}{c}N_{T}=16 \times 16 \\
N_{R}=4 \\
N_{U}=4\end{array}$ & $\begin{array}{c}\text { ABF: steering vector } \\
\text { DBF: ZF }\end{array}$ & $10 @ S N R 0$ dB \\
\hline
\end{tabular}

\section{CONCLUSION}

A new user selection algorithm is proposed for BDMA scheme of massive MIMO system in this work. Based on deploying Zero-Forcing and new schedule in hybrid beamforming, the multiuser interference (MUI) is significantly reduced when we increase the number of BS antenna. Simulated results confirmed that proposed solution can attain spectrum efficiency (SE) as good as only digital beamforming.

\section{ACKNOWLEDGEMENTS}

Hong Son $\mathrm{Vu}$ was funded by Vingroup Joint Stock Company and supported by the Domestic Master/Ph.D. Scholarship Programme of Vingroup Innovation Foundation (VINIF), Vingroup Big Data Institute (VINBIGDATA), code VINIF.2020.ThS.66.

\section{REFERENCES}

[1] C. Sun, X. Gao, S. Jin, M. Matthaiou, Z. Ding, and C. Xiao, "Beam division multiple access transmission for massive MIMO communications," in IEEE Transactions on Communications, vol. 63, no. 6, pp. 2170-2184, Jun. 2015, doi: 10.1109/TCOMM.2015.2425882.

[2] Z. Jiang, S. Chen, S. Zhou, and Z. Niu, "Joint user scheduling and beam selection optimization for beam-based massive MIMO downlinks," in IEEE Transactions on Wireless Communications, vol. 17, no. 4, pp. 2190-2204, Apr. 2018, doi: 10.1109/TWC.2018.2789895

[3] J. Salz and J. H. Winters, "Effect of fading correlation on adaptive arrays in digital mobile radio," in IEEE Transactions on Vehicular Technology, vol. 43, no. 4, pp. 1049-1057, Nov. 1994, doi: 10.1109/25.330168

[4] M. Sharif and B. Hassibi, "On the capacity of MIMO broadcast channels with partial side information," in IEEE Transactions on Information Theory, vol. 51, no. 2, pp. 506-522, Feb. 2005, doi: 10.1109/TIT.2004.840897.

[5] G. J. Foschini and M. J. Gans, "On limits of wireless communications in a fading environment when using multiple antennas," in Wireless Personal Communications, vol. 6, no. 3, pp. 311-335, 1998, doi: 10.1023/A:1008889222784.

[6] P. Varzakas, "Average channel capacity for Rayleigh fading spread spectrum MIMO systems," in International Journal Communication Systems, vol. 19, no. 10, pp. 1081-1087, Feb. 2006, doi: 10.1002/dac.784

[7] Da-Shan Shiu, G. J. Foschini, M. J. Gans, and J. M. Kahn, "Fading correlation and its effect on the capacity of multielement antenna systems," in IEEE Transactions on Communications, vol. 48, no. 3, pp. 502-513, Mar. 2000, doi: 10.1109/26.837052.

[8] T. Lin, J. Cong, Y. Zhu, J. Zhang, and K. Ben Letaief, "Hybrid beamforming for millimeter wave systems using the MMSE criterion," in IEEE Transactions on Communications, vol. 67, no. 5, pp. 3693-3708, May 2019, doi: 10.1109/TCOMM.2019.2893632.

[9] A. A. Nasir, H. D. Tuan, T. Q. Duong, H. V. Poor, and L. Hanzo, "Hybrid beamforming for multi-user millimeter-wave networks," in IEEE Transactions on Vehicular Technology, vol. 69, no. 3, pp. 2943-2956, Mar. 2020, doi: 10.1109/TVT.2020.2966122.

[10] M. N. Kulkarni, A. Ghosh, and J. G. Andrews, "A comparison of MIMO techniques in downlink millimeter wave cellular networks with hybrid beamforming," in IEEE Transactions on Communications, vol. 64, no. 5, pp. 1952-1967, May 2016, doi: 10.1109/TCOMM.2016.2542825.

[11] K. Satyanarayana, M. El-Hajjar, P. Kuo, A. Mourad, and L. Hanzo, "Dual-function hybrid beamforming and transmit diversity aided millimeter wave architecture," in IEEE Transactions on Vehicular Technology, vol. 67, no. 3, pp. 2798-2803, Mar. 2018, doi: 10.1109/TVT.2017.2737782. 
[12] A. Garcia-Rodriguez, V. Venkateswaran, P. Rulikowski, and C. Masouros, "Hybrid analog-digital precoding revisited under realistic RF modeling," in IEEE Wireless Communications Letters, vol. 5, no. 5, pp. 528-531, Oct. 2016, doi: 10.1109/LWC.2016.2598777.

[13] A. Alkhateeb, O. El Ayach, G. Leus, and R. W. Heath, "Channel estimation and hybrid precoding for millimeter wave cellular systems," in IEEE Journal of Selected Topics in Signal Processin, vol. 8, no. 5, pp. 831-846, Oct. 2014, doi: 10.1109/JSTSP.2014.2334278.

[14] G. Caire and S. Shamai, "On the achievable throughput of a multiantenna Gaussian broadcast channel," in IEEE Transactions on Information Theory, vol. 49, no. 7, pp. 1691-1706, Jul. 2003, doi: 10.1109/TIT.2003.813523.

[15] Q. H. Spencer and M. Haardt, "Capacity and downlink transmission algorithms for a multi-user MIMO channel," Conference Record of the Thirty-Sixth Asilomar Conference on Signals, Systems and Computers, 2002, 2002, pp. 1384-1388 vol. 2, doi: 10.1109/ACSSC.2002.1197006.

[16] Z. Tu and R. S. Blum, "Multiuser diversity for a dirty paper approach," in IEEE Communications Letters, vol. 7, no. 8, pp. 370-372, Aug. 2003, doi: 10.1109/LCOMM.2003.815652.

[17] S. Vishwanath, N. Jindal, and A. Goldsmith, "Duality, achievable rates, and sum-rate capacity of Gaussian MIMO broadcast channels," in IEEE Transactions on Information Theory, vol. 49, no. 10, pp. 2658-2668, Oct. 2003, doi: 10.1109/TIT.2003.817421.

[18] P. Viswanath and D. N. C. Tse, "Sum capacity of the vector Gaussian broadcast channel and uplink-downlink duality," in IEEE Transactions on Information Theory, vol. 49, no. 8, pp. 1912-1921, Aug. 2003, doi: 10.1109/TIT.2003.814483.

[19] H. Viswanathan, S. Venkatesan, and H. Huang, "Downlink capacity evaluation of cellular networks with known-interference cancellation," in IEEE Journal on Selected Areas in Communications, vol. 21, no. 5, pp. 802-811, Jun. 2003, doi: 10.1109/JSAC.2003.810346.

[20] G. Dimic and N. D. Sidiropoulos, "On downlink beamforming with greedy user selection: performance analysis and a simple new algorithm," in IEEE Transactions on Signal Processing, vol. 53, no. 10, pp. 3857-3868, Oct. 2005, doi: 10.1109/TSP.2005.855401.

[21] T. Yoo and A. Goldsmith, "On the optimality of multiantenna broadcast scheduling using zero-forcing beamforming," in IEEE Journal on Selected Areas in Communications, vol. 24, no. 3, pp. 528-541, Mar. 2006, doi: 10.1109/JSAC.2005.862421.

[22] S. Ozyurt and M. Torlak, "Performance analysis of optimum zero-forcing beamforming with greedy user selection," in IEEE Communications Letters, vol. 16, no. 4, pp. 446-449, Apr. 2012, doi: 10.1109/LCOMM.2012.022112.112036.

[23] R. de Francisco, M. Kountouris, D. T. M. Slock, and D. Gesbert, "Orthogonal linear beamforming in MIMO broadcast channels," 2007 IEEE Wireless Communications and Networking Conference, 2007, pp. 1210-1215, doi: 10.1109/WCNC.2007.229.

[24] J. Duplicy, D. P. Palomar, and L. Vandendorpe, "Adaptive orthogonal beamforming for the mimo broadcast channel," 2007 2nd IEEE International Workshop on Computational Advances in Multi-Sensor Adaptive Processing, 2007, pp. 77-80, doi: 10.1109/CAMSAP.2007.4497969.

[25] C. Zhang, W. Xu, and M. Chen, "Hybrid zero-forcing beamforming/orthogonal beamforming with user selection for MIMO broadcast channels," in IEEE Communications Letters, vol. 13, no. 1, pp. 10-12, Jan. 2009, doi: 10.1109/LCOMM.2009.081466.

[26] C. G. Tsinos, S. Maleki, S. Chatzinotas, and B. Ottersten, "On the energy-efficiency of hybrid analog-digital transceivers for single- and multi-carrier large antenna array systems," in IEEE Journal on Selected Areas in Communications, vol. 35, no. 9, pp. 1980-1995, Sept. 2017, doi: 10.1109/JSAC.2017.2720918.

[27] T. S. Rappaport, R. W. Heath, R. C. Daniels, and J. N. Murdock, "Millimeter wave wireless communications," 1st Ed., Pearson, 2014

[28] L. Liang, W. Xu, and X. Dong, "Low-complexity hybrid precoding in massive multiuser MIMO systems," in IEEE Wireless Communications Letters, vol. 3, no. 6, pp. 653-656, Dec. 2014, doi: 10.1109/LWC.2014.2363831.

[29] O. E. Ayach, R. W. Heath, S. Abu-Surra, S. Rajagopal, and Z. Pi, "Low complexity precoding for large millimeter wave MIMO systems," 2012 IEEE International Conference on Communications (ICC), 2012, pp. 3724-3729, doi: 10.1109/ICC.2012.6363634

[30] J. Singh and S. Ramakrishna, "On the feasibility of codebook-based beamforming in millimeter wave systems with multiple antenna arrays," in IEEE Transactions on Wireless Communications, vol. 14, no. 5, pp. 2670-2683, May 2015, doi: 10.1109/TWC.2015.2390637.

[31] J. Jing, C. Xiaoxue, and X. Yongbin, "Energy-efficiency based downlink multi-user hybrid beamforming for millimeter wave massive MIMO system," in Journal China University Posts Telecommunication, vol. 23, no. 4, pp. 53-62, Aug. 2016, doi: 10.1016/S1005-8885(16)60045-6.

[32] A. Alkhateeb, G. Leus, and R. W. Heath, "Limited feedback hybrid precoding for multi-user millimeter wave systems," in IEEE Transactions on Wireless Communications, vol. 14, no. 11, pp. 6481-6494, Nov. 2015, doi: 10.1109/TWC.2015.2455980.

\section{BIOGRAPHIES OF AUTHORS}

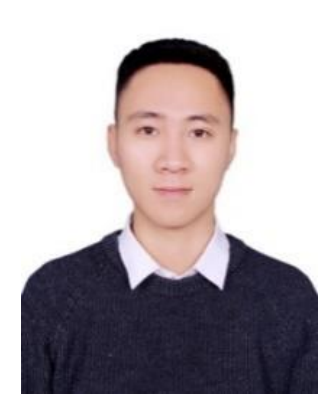

Hong Son Vu (D) SC P received his B.S (2019) and M. Sc (2021) degree in Electrical Engineering from Hanoi University of Science and Technology. He is currently a research assistant at Department of Instrumentation and Industrial Informatic (3I), School of Electrical Engineering (SEE), Hanoi University of Science and Technology (HUST). His current interests include signal processing and massive MIMO system. He can be contacted at email: hongsonvu1996@gmail.com. 

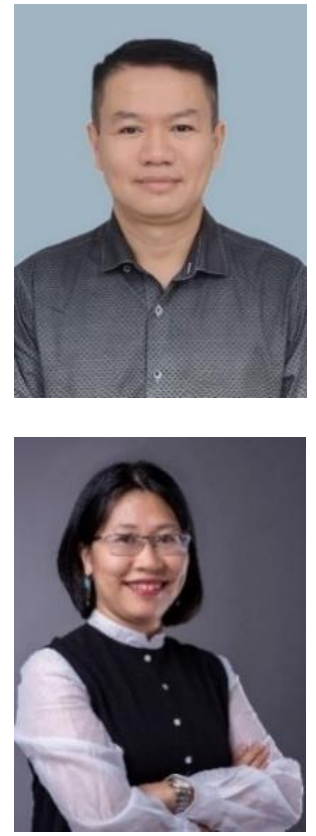

Kien Trung Truong (iD $\mathrm{SC}$ P earned his B.S. in Electronics and Telecommunications from Hanoi University of Science and Technology in Hanoi, Vietnam, in 2002, and his M.Sc. and Ph.D. in Electrical Engineering from the University of Texas at Austin in Austin, Texas, USA, in 2008 and 2012. He is currently a lecturer in engineering at Fulbright University Vietnam, Ho Chi Minh City, Vietnam. His current research interests include massive MIMO communications, millimeter-wave communications, and wireless energy harvesting. He was co-recipient of the 2013 EURASIP Journal on Wireless Communications and Networking Best Paper Award and the 2014 IEEE/KICS Journal of Communications and Networks Best Paper Award. He was a fellow of the Vietnam Education Foundation in 2006. He can be contacted at email: kien.truong@ fulbright.edu.vn.

Minh Thuy Le (D) IS SC P graduated from Hanoi University of Science and Technology with a bachelor's degree in electrical engineering in 2006, a master's degree in electrical engineering in 2008, and Ph.D. (2013) degree in Optique and Radio Frequency from Grenoble Institute of Technology, France. She is a lecturer, as well as the group leader of the Radio Frequency group at the Department of Instrumentation and Industrial Informatics (3I), School of Electrical Engineering (SEE), Hanoi University of Science and Technology (HUST). Her current interests include built-in antennas, antenna arrays, beamforming, metamaterials, indoor localization and RF energy harvesting, wireless power transfer, autonomous wireless sensor. She can be contacted at email: thuy.leminh@hust.edu.vn. 